International Journal of Pure and Applied Mathematics

Volume 105 No. 4 2015, 835-852

ISSN: 1311-8080 (printed version); ISSN: 1314-3395 (on-line version)

url: http://www.ijpam.eu

doi: http://dx.doi.org/10.12732/ijpam.v105i4.22

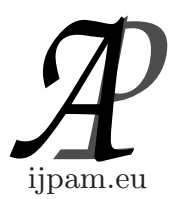

\title{
THE $\gamma$-SPECTRUM OF CYCLE WITH ONE CHORD
}

\author{
Varanoot Khemmani ${ }^{1}$, Supaporn Saduakdee ${ }^{2} \S$ \\ ${ }^{1}$ Department of Mathematics \\ Srinakharinwirot University \\ Sukhumvit 23, Bangkok, 10110, THAILAND
}

\begin{abstract}
Let $G$ be a graph of order $n$ and size $m$. A $\gamma$-labeling of $G$ is a one-to-one function $f: V(G) \rightarrow\{0,1,2, \ldots, m\}$ that induces an edge-labeling $f^{\prime}: E(G) \rightarrow\{1,2, \ldots, m\}$ on $G$ defined by $f^{\prime}(e)=|f(u)-f(v)|$ for each edge $e=u v$ of $G$. The value of $f$ is defined as
\end{abstract}

$$
\operatorname{val}(f)=\sum_{e \in E(G)} f^{\prime}(e) .
$$

The $\gamma$-spectrum of a graph $G$ is defined as

$$
\operatorname{spec}(G)=\{\operatorname{val}(f): f \text { is a } \gamma \text {-labeling of } G\} .
$$

In this paper, $\gamma$-spectrum of cycle with one chord is determined.

\section{AMS Subject Classification: $05 \mathrm{C} 78$}

Key Words: $\gamma$-labeling, $\gamma$-spectrum, cycle with one chord

\section{Introduction}

In 2005 Chartrand, Erwin, VanderJagt, and Zhang [2] studied, for a graph $G$ of order $n$ and size $m$, a $\gamma$-labeling of $G$ defined as a one-to-one function $f: V(G) \rightarrow\{0,1,2, \ldots, m\}$ that induces a labeling $f^{\prime}: E(G) \rightarrow\{1,2, \ldots, m\}$

Received: October 15, 2015

(C) 2015 Academic Publications, Ltd.

${ }^{\S}$ Correspondence author url: www.acadpubl.eu 
of the edges of $G$ defined by $f^{\prime}(e)=|f(u)-f(v)|$ for each edge $e=u v$ of $G$. Therefore, a graph $G$ of order $n$ and size $m$ has a $\gamma$-labeling if and only if $m \geq n-1$. Each $\gamma$-labeling $f$ is assigned a value denoted by $\operatorname{val}(f)$ and defined by

$$
\operatorname{val}(f)=\sum_{e \in E(G)} f^{\prime}(e) .
$$

Obviously, since $f$ is one-to-one, it follows that $f^{\prime}(e) \geq 1$, for any edge $e$, and therefore, $\operatorname{val}(f) \geq m$. Moreover, $G$ has a $\gamma$-labeling if and only if $m \geq n-1$ and every connected graph has a $\gamma$-labeling.

Figure 1 shows nine $\gamma$-labelings $f_{1}, f_{2}, \ldots, f_{9}$ of the path $P_{5}$ of order 5 (where the vertex labels are shown above each vertex and the induced edge labels are shown below each edge). The value of each $\gamma$-labeling is shown in Figure 1 as well.

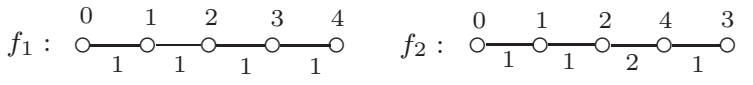

$$
\begin{aligned}
& \operatorname{val}\left(\mathrm{f}_{1}\right)=4 \\
& \operatorname{val}\left(\mathrm{f}_{2}\right)=5
\end{aligned}
$$

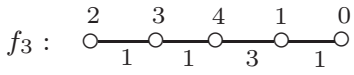

$$
\begin{aligned}
& \operatorname{val}\left(\mathbf{f}_{3}\right)=6
\end{aligned}
$$

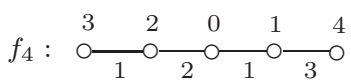

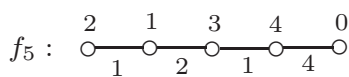

$$
\begin{aligned}
& f_{6}: \stackrel{0}{\circ} \underset{3}{\circ} \stackrel{3}{\circ} \stackrel{2}{\circ} \underset{2}{\circ} \stackrel{4}{\circ} \stackrel{1}{\circ} \\
& \operatorname{val}\left(\mathrm{f}_{4}\right)=7 \\
& \operatorname{val}\left(f_{5}\right)=8 \\
& \operatorname{val}\left(\mathbf{f}_{6}\right)=9
\end{aligned}
$$

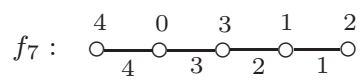

$$
\begin{aligned}
& f_{8}: \stackrel{2}{\circ} \underset{2}{\circ} \stackrel{0}{\circ} \stackrel{3}{\circ} \frac{1}{2} \underset{3}{\circ} \\
& \operatorname{val}\left(\mathbf{f}_{8}\right)=10 \\
& f_{9}: \quad \stackrel{3}{\circ} \longrightarrow_{3} \stackrel{0}{\circ} \underset{4}{\circ} \underset{3}{\circ} \stackrel{1}{\circ}{ }_{1}^{2} \\
& \operatorname{val}\left(f_{9}\right)=11
\end{aligned}
$$

Figure 1: Some $\gamma$-labelings of $P_{5}$

If the induced edge-labeling $f^{\prime}$ of a $\gamma$-labeling $f$ of a graph is also one-to-one, then $f$ is a graceful labeling. Among all labelings of graphs, graceful labelings are probably the best known and most studied. Graceful labelings originated with a paper of Rosa [11], who used the term $\beta$-valuations. A few years later, Golomb [9] called these labelings "graceful" and this is the terminology that has been used since then. Gallian [8] has written an extensive survey on labelings of graphs.

The maximum value and the minimum value of a $\gamma$-labeling of $G$ are defined in $[2]$ as

$$
\operatorname{val}_{\text {max }}(G)=\max \{\operatorname{val}(f): f \text { is a } \gamma \text {-labeling of } G\}
$$


and

$$
\operatorname{val}_{\text {min }}(G)=\min \{\operatorname{val}(f): f \text { is a } \gamma \text {-labeling of } G\},
$$

respectively. It turns out that $\operatorname{val}_{\max }\left(P_{5}\right)=11$ and $\operatorname{val}_{\min }\left(P_{5}\right)=4$.

A $\gamma$-labeling $g$ of $G$ is a $\gamma$-max labeling if $\operatorname{val}(g)=\operatorname{val}_{\max }(G)$ and a $\gamma$-labeling $h$ is a $\gamma$-min labeling if $\operatorname{val}(h)=\operatorname{val}_{\text {min }}(G)$.

The subject of maximum and minimum values of a $\gamma$-labeling of path $P_{n}$, cycle $C_{n}$, complete graph $K_{n}$, double star $S_{p, q}$ and complete bipartite graph $K_{r, s}$ and cycle with a triangle $C_{n}^{\triangle}$ are studied in $[1,2,3,5,6]$.

Later, the extreme values of $\gamma$-labeling of cycle with one chord $C_{n}+e$, i.e., cycle with a chord $e$ joining two nonadjacent vertices in cycle $C_{n}$, are established in [10] as we state next.

Theorem 1.1 ([10]). Let $C_{n}+e$ be a cycle with one chord of order $n \geq 4$. Then we have the following.

(a) $\operatorname{val}_{\min }\left(C_{n}+e\right)=2 n-1$.

(b) For an odd integer $n$, $\operatorname{val}_{\max }\left(C_{n}+e\right)=\frac{n^{2}+6 n-3}{2}$.

(c) For an even integer $n$,

(i) $\operatorname{val}_{\max }\left(C_{n}+e\right)=\frac{n^{2}+6 n+2}{2}$ where $e$ is a chord joining two vertices with odd distance in even cycle $C_{n}$,

(ii) $\operatorname{val}_{\max }\left(C_{n}+e\right)= \begin{cases}\frac{n^{2}+5 n-2}{2} & \text { if } n=4,6,8 \\ \frac{n^{2}+6 n-10}{2} & \text { if } n \geq 8\end{cases}$

where $e$ is a chord joining two vertices with even distance in even cycle $C_{n}$.

The $\gamma$-spectrum of a graph $G$ is defined in [2] as

$$
\operatorname{spec}(G)=\{\operatorname{val}(f): f \text { is a } \gamma \text {-labeling of } G\} .
$$

Observe that $\operatorname{val}_{\min }(G), \operatorname{val}_{\max }(G) \in \operatorname{spec}(G)$ for every graph $G$. For integers $a$ and $b$ with $a<b$, let

$$
[a, b]=\{a, a+1, \ldots, b\}
$$

be a consecutive set of integers between $a$ and $b$. Moreover, for even integers $a$ and $b$ with $a<b$, let 


$$
E[a, b]=\{a, a+2, a+4, \ldots, b\}
$$

be an even consecutive set of integers between $a$ and $b$.

Thus for every graph $G, \operatorname{spec}(G) \subseteq\left[\operatorname{val}_{\min }(G), \operatorname{val}_{\max }(G)\right]$. The $\gamma$-spectra of stars, paths, cycles and complete graphs are determined in $[2,7]$. Next we recall the $\gamma$-spectrum of $C_{n}$ for each integer $n \geq 3$.

Theorem $1.2([7])$. For each integer $n \geq 3$,

$$
\operatorname{spec}\left(C_{n}\right)=E\left[\operatorname{val}_{\min }\left(C_{n}\right), \operatorname{val}_{\max }\left(C_{n}\right)\right] .
$$

Fonseca, Saenpholphat, and Zhang [5] suggest one to determine the $\gamma$ spectrum of $C_{n}^{\triangle}$. In this paper, we study not only the $\gamma$-spectrum of cycle with a triangle $C_{n}^{\triangle}$ but also the $\gamma$-spectrum of cycle with one chord $C_{n}+e$ in order to make broader generalization. Observe that in Theorem 1.2, the value of any $\gamma$-labeling of cycle is always even. We show that the value of any $\gamma$-labeling of cycle with one chord is not the case.

The reader is referred to Chartrand and Zhang [4] for basic definitions and terminology not mentioned here.

\section{Main Result}

In this section we show that the $\gamma$-spectrum of $C_{n}+e$ is a consecutive set of integers between $\operatorname{val}_{\min }\left(C_{n}+e\right)$ and $\operatorname{val}_{\max }\left(C_{n}+e\right)$, in the main theorem as follows.

Theorem 2.1. For every integer $n \geq 4$,

$$
\operatorname{spec}\left(C_{n}+e\right)=\left[\operatorname{val}_{\min }\left(C_{n}+e\right), \operatorname{val}_{\max }\left(C_{n}+e\right)\right] .
$$

Throughout this main section, let $C_{n}+e$ be a cycle with one chord of order $n$ which is obtained from

a cycle $C_{n}: v_{1}, v_{2}, \ldots, v_{r-1}, v_{r}, v_{r+1}, \ldots, v_{n}, v_{1}$ and a chord $e=v_{1} v_{r}$,

where $3 \leq r \leq n-1$.

Since $C_{n}+v_{1} v_{r}$ is isomorphic to $C_{n}+v_{1} v_{n-r+2}$ for each $r$ with $3 \leq r \leq n-1$, it is sufficient to determine that for each $r$ with $3 \leq r \leq\left\lceil\frac{n+1}{2}\right\rceil$,

$$
\operatorname{spec}\left(C_{n}+v_{1} v_{r}\right)=\left[\operatorname{val}_{\text {min }}\left(C_{n}+v_{1} v_{r}\right), \operatorname{val}_{\text {max }}\left(C_{n}+v_{1} v_{r}\right)\right] .
$$


Observe that Theorem 1.1 provides that

$$
\begin{aligned}
\operatorname{val}_{\text {min }}\left(C_{n}+v_{1} v_{r}\right) & =(2 n-2)+1 \\
\operatorname{val}_{\text {max }}\left(C_{n}+v_{1} v_{r}\right) & =(2 n-2)+\left|\left[\operatorname{val}_{\text {min }}\left(C_{n}+v_{1} v_{r}\right), \operatorname{val}_{\text {max }}\left(C_{n}+v_{1} v_{r}\right)\right]\right| .
\end{aligned}
$$

Therefore our goal is to find a $\gamma$-labeling $f_{l}$ of $C_{n}+v_{1} v_{r}$ with $\operatorname{val}\left(f_{l}\right)=$ $(2 n-2)+l$ for each integer $l$ with

$$
1 \leq l \leq\left|\left[\operatorname{val}_{\min }\left(C_{n}+v_{1} v_{r}\right), \operatorname{val}_{\max }\left(C_{n}+v_{1} v_{r}\right)\right]\right|
$$

The following propositions will be used to deduce a $\gamma$-labeling $f_{l}$ of $C_{n}+v_{1} v_{r}$ for achieving the main result.

Proposition 2.2. For every even integer $n \geq 6$,

$$
\operatorname{spec}\left(C_{n}+e\right)=\left[\operatorname{val}_{\min }\left(C_{n}+e\right), \operatorname{val}_{\max }\left(C_{n}+e\right)\right]=\left[2 n-1, \frac{n^{2}+6 n+2}{2}\right]
$$

where $e$ is a chord joining two vertices with odd distance in even cycle $C_{n}$.

Proof. By (1), we may assume that $C_{n}+e=C_{n}+v_{1} v_{r}$ where $3 \leq r \leq \frac{n}{2}+1$ and $r$ is even.

For each integer $i$ with $0 \leq i \leq \frac{n}{2}-1$, let

$$
\Delta_{i}= \begin{cases}2 n+2 & \text { if } i=0 \\ 2 n-4 i-1 & \text { if } 1 \leq i \leq \frac{n}{2}-2 \\ \frac{n}{2}+3 & \text { if } i=\frac{n}{2}-1\end{cases}
$$

We will show that, for each integer $l$ with

$$
1 \leq l \leq \sum_{i=0}^{\frac{n}{2}-1} \Delta_{i}=\left|\left[\operatorname{val}_{\text {min }}\left(C_{n}+v_{1} v_{r}\right), \operatorname{val}_{\text {max }}\left(C_{n}+v_{1} v_{r}\right)\right]\right|,
$$

there exists a $\gamma$-labeling $f_{l}$ whose value is $(2 n-2)+l$.

First, we consider $1 \leq l \leq \Delta_{0}$ as follows: 
I. For $1 \leq l \leq 8$, we can define a $\gamma$-labeling $f_{l}$ of $C_{n}+v_{1} v_{r}$ by

$$
\begin{aligned}
& f_{l}\left(v_{1}\right)= \begin{cases}r-3 & \text { if } l=2,8 \\
r-2 & \text { if } l=1,4,7 \\
r-1 & \text { if } l=3,6 \\
r & \text { if } l=5\end{cases} \\
& f_{l}\left(v_{i}\right)= \begin{cases}r-i-1 & \text { if } 2 \leq i \leq r-2 \text { and } l=1,3,4,5,6,7 \\
r-i-2 & \text { if } 2 \leq i \leq r-2 \text { and } l=2,8\end{cases} \\
& f_{l}\left(v_{r-1}\right)= \begin{cases}0 & \text { if } l=1,3,4,5,6,7 \\
r-2 & \text { if } l=2,8\end{cases} \\
& f_{l}\left(v_{r}\right)= \begin{cases}r-1 & \text { if } l=1,2 \\
r & \text { if } l=3,4 \\
r+1 & \text { if } l=5,6,7,8\end{cases} \\
& f_{l}\left(v_{i}\right)=f_{l}\left(v_{r}\right)+i-r \text { if } r+1 \leq i \leq n \text { and } 1 \leq l \leq 8 .
\end{aligned}
$$

If $l=1,3,4,5,6$ and 7 , then

$$
\operatorname{val}\left(f_{l}\right)=-f_{l}\left(v_{1}\right)-2 f_{l}\left(v_{r-1}\right)+f_{l}\left(v_{r}\right)+2 f_{l}\left(v_{n}\right)=(2 n-2)+l .
$$

If $l=2$ and 8 , then

$$
\operatorname{val}\left(f_{l}\right)=-f_{l}\left(v_{1}\right)-2 f_{l}\left(v_{r-2}\right)+f_{l}\left(v_{r}\right)+2 f_{l}\left(v_{n}\right)=(2 n-2)+l .
$$

II. For $9 \leq l \leq 2 r+2$, let $f_{l}$ be a $\gamma$-labeling of $C_{n}+v_{1} v_{r}$ defined by

$$
\begin{aligned}
& f_{l}\left(v_{1}\right)= \begin{cases}r-2 & \text { if } l \text { is even } \\
r-1 & \text { if } l \text { is odd }\end{cases} \\
& f_{l}\left(v_{i}\right)= \begin{cases}i-2 & \text { if } 2 \leq i \leq r-1 \\
r+2 & \text { if } i=r \\
i+2 & \text { if } r+1 \leq i \leq\left\lceil\frac{l}{2}\right\rceil+r-5 \\
r+1 & \text { if } i=\left\lceil\frac{l}{2}\right\rceil+r-4 \\
i+1 & \text { if }\left\lceil\frac{l}{2}\right\rceil+r-3 \leq i \leq n .\end{cases}
\end{aligned}
$$


If $l=9$ and 10 , then

$$
\operatorname{val}\left(f_{l}\right)=-f_{l}\left(v_{1}\right)-2 f_{l}\left(v_{2}\right)+3 f_{l}\left(v_{r}\right)-2 f_{l}\left(v_{r+1}\right)+2 f_{l}\left(v_{n}\right)=(2 n-2)+l .
$$

If $11 \leq l \leq 2 r+2$, then

$$
\begin{aligned}
\operatorname{val}\left(f_{l}\right)= & -f_{l}\left(v_{1}\right)-2 f_{l}\left(v_{2}\right)+f_{l}\left(v_{r}\right)+2 f_{l}\left(v_{\left\lceil\frac{l}{2}\right\rceil+r-5}\right)-2 f_{l}\left(v_{\left\lceil\frac{l}{2}\right\rceil+r-4}\right) \\
& +2 f_{l}\left(v_{n}\right) \\
= & (2 n-2)+l .
\end{aligned}
$$

III. For $2 r+3 \leq l \leq \Delta_{0}=2 n+2$, define a $\gamma$-labeling $f_{l}$ of $C_{n}+v_{1} v_{r}$ by

$$
f_{l}\left(v_{i}\right)= \begin{cases}\left\lceil\frac{l-5}{2}\right\rceil & \text { if } i=1 \\ 0 & \text { if } i=2 \\ \left\lceil\frac{l-6}{2}\right\rceil-r+i & \text { if } 3 \leq i \leq r-1 \\ \left\lceil\frac{l}{2}\right\rceil-r+i & \text { if } r \leq i \leq n+r-\left\lceil\frac{l+2}{2}\right\rceil \\ \left\lceil\frac{l-2}{2}\right\rceil-n-r+i & \text { if } n+r-\left\lceil\frac{l+2}{2}\right\rceil+1 \leq i \leq n .\end{cases}
$$

If $2 r+3 \leq l \leq 2 n$, then

$$
\begin{aligned}
\operatorname{val}\left(f_{l}\right) & =f_{l}\left(v_{1}\right)-2 f_{l}\left(v_{2}\right)+f_{l}\left(v_{r}\right)+2 f_{l}\left(v_{n+r-\left\lceil\frac{l}{2}\right\rceil+1}\right)-2 f_{l}\left(v_{n+r-\left\lceil\frac{l}{2}\right\rceil+2}\right) \\
& =(2 n-2)+l .
\end{aligned}
$$

If $l=2 n+1$ and $2 n+2$, then

$$
\operatorname{val}\left(f_{l}\right)=f_{l}\left(v_{1}\right)-2 f_{l}\left(v_{2}\right)+3 f_{l}\left(v_{r}\right)-2 f_{l}\left(v_{r+1}\right)=(2 n-2)+l .
$$

Next, we consider $\Delta_{0}+1 \leq l \leq \sum_{i=0}^{\frac{n}{2}-1} \Delta_{i}$, by letting $\left(t, l_{t}\right)$ be a pair of integers with $1 \leq t \leq \frac{n}{2}-1$ and $l_{t}=l-\sum_{i=0}^{t-1} \Delta_{i}$. Certainly, $1 \leq l_{t} \leq \Delta_{t}$. We construct a $\gamma$-labeling $f_{l}$ as the following procedure:

IV. For $\Delta_{0}+1 \leq l \leq \sum_{i=0}^{\frac{n-r}{2}-1} \Delta_{i}$, define a $\gamma$-labeling $f_{l}$ of $C_{n}+v_{1} v_{r}$ by

$$
f_{l}\left(v_{1}\right)= \begin{cases}n-(t-1) & \text { if } l_{t}=\Delta_{t}-4 \\ n-t & \text { if } l_{t} \text { is even and } l_{t} \neq \Delta_{t}-3, \Delta_{t}-1 \\ n-(t+1) & \text { if } l_{t} \text { is odd and } l_{t} \neq \Delta_{t}-4 \\ n-(t+2) & \text { if } l_{t}=\Delta_{t}-3, \Delta_{t}-1\end{cases}
$$




$$
\begin{aligned}
& f_{l}\left(v_{2}\right)=0 \\
& f_{l}\left(v_{i}\right)= \begin{cases}n+i-(t+r+1) & \text { if } 3 \leq i \leq r-1 \\
& \text { and } 1 \leq l_{t} \leq \Delta_{t}-(2 r-1) \\
n+i-(t+r+2) & \text { if } 3 \leq i \leq f_{l}\left(v_{2 t+r}\right)+t+r-n+1 \\
n+i-(t+r+1) & \text { if } f_{l}\left(v_{2 t+r}\right)+t+r-n+2 \leq i \leq r-1 \\
& \text { and } \Delta_{t}-(2 r-2) \leq l_{t} \leq \Delta_{t}-7 \\
n+i-(t+r+2) & \text { if } 3 \leq i \leq r-1 \text { and } \Delta_{t}-6 \leq l_{t} \leq \Delta_{t}\end{cases} \\
& f_{l}\left(v_{i}\right)= \begin{cases}\frac{i-r+1}{2} & \text { if } i \text { is odd and } r \leq i \leq 2 t+r+1 \\
n+1-\left(\frac{i-r}{2}\right) & \text { if } i \text { is even and } r \leq i \leq 2 t+r-2\end{cases} \\
& f_{l}\left(v_{2 t+r}\right)= \begin{cases}\left\lceil\frac{l_{t}+2}{2}\right\rceil+t & \text { if } 1 \leq l_{t} \leq \Delta_{t}-5 \\
\left\lceil\frac{l_{t}}{2}\right\rceil+t & \text { if } l_{t}=\Delta_{t}-4 \\
\left\lceil\frac{l_{t}+3}{2}\right\rceil+t & \text { if } \Delta_{t}-3 \leq l_{t} \leq \Delta_{t}\end{cases} \\
& f_{l}\left(v_{i}\right)= \begin{cases}i-(t+r-1) & \text { if } 2 t+r+2 \leq i \leq n \text { and } l_{t}=1,2 \\
i-(t+r) & \text { if } 2 t+r+2 \leq i \leq f_{l}\left(v_{2 t+r}\right)+t+r-1 \\
& \text { and } 3 \leq l_{t} \leq \Delta_{t}-(2 r+1) \\
i-(t+r-1) & \text { if } f_{l}\left(v_{2 t+r}\right)+t+r \leq i \leq n \\
& \text { and } 3 \leq l_{t} \leq \Delta_{t}-(2 r+1) \\
i-(t+r) & \text { if } 2 t+r+2 \leq i \leq n \\
& \text { and } \Delta_{t}-2 r \leq l_{t} \leq \Delta_{t} .\end{cases}
\end{aligned}
$$

Then

$$
\begin{aligned}
\operatorname{val}\left(f_{l}\right)= & f_{l}\left(v_{1}\right)-2 f_{l}\left(v_{2}\right)+3 f_{l}\left(v_{r}\right) \underset{r+1 \leq i \leq 2 t+r+1}{-2 \sum_{i \text { is odd }} f_{l}\left(v_{i}\right)} \underset{\substack{r+2 \leq i \leq 2 t+r-2 \\
i \text { is even }}}{2 \sum_{l} f_{l}\left(v_{i}\right)} \\
& +2 f_{l}\left(v_{2 t+r}\right) \\
= & 2 n t+n-2 t^{2}-1+f_{l}\left(v_{1}\right)+2 f_{l}\left(v_{2 t+r}\right)=(2 n-2)+l .
\end{aligned}
$$


V. For $\sum_{i=0}^{\frac{n-r}{2}-1} \Delta_{i}+1 \leq l \leq \sum_{i=0}^{\frac{n}{2}-3} \Delta_{i}$, now we construct a $\gamma$-labeling $f_{l}$ of $C_{n}+v_{1} v_{r}$ by

$$
f_{l}\left(v_{1}\right)= \begin{cases}n-(t-1) & \text { if } l_{t}=\Delta_{t}-4 \\ n-t & \text { if } l_{t} \text { is even and } l_{t} \neq \Delta_{t}-3, \Delta_{t}-1 \\ n-(t+1) & \text { if } l_{t} \text { is odd and } l_{t} \neq \Delta_{t}-4 \\ n-(t+2) & \text { if } l_{t}=\Delta_{t}-3, \Delta_{t}-1\end{cases}
$$$$
f_{l}\left(v_{2}\right)=0
$$$$
f_{l}\left(v_{i}\right)= \begin{cases}\frac{n-2+i-r}{2} & \text { if } i \text { is even and } 3 \leq i \leq 2 t+r-n+4 \\ \frac{n+5+r-i}{2} & \text { if } i \text { is odd and } 3 \leq i \leq 2 t+r-n+1\end{cases}
$$$$
f_{l}\left(v_{2 t+r-n+3}\right)= \begin{cases}\left\lceil\frac{l_{t}+2}{2}\right\rceil+t & \text { if } 1 \leq l_{t} \leq \Delta_{t}-5 \\ \left\lceil\frac{l_{t}}{2}\right\rceil+t & \text { if } l_{t}=\Delta_{t}-4 \\ \left\lceil\frac{l_{t}+3}{2}\right\rceil+t & \text { if } \Delta_{t}-3 \leq l_{t} \leq \Delta_{t}\end{cases}
$$

$$
\begin{aligned}
& f_{l}\left(v_{i}\right)= \begin{cases}n+i-(t+r+1) & \text { if } 2 t+r-n+5 \leq i \leq r-1 \\
& \text { and } 1 \leq l_{t} \leq 4 \\
n+i-(t+r+2) & \text { if } 2 t+r-n+5 \leq i \leq f_{l}\left(v_{2 t+r-n+3}\right) \\
& \\
& \text { and } 5 \leq l_{t} \leq \Delta_{t}-7 \quad+t+n+1 \\
n+i-(t+r+1) & \text { if } f_{l}\left(v_{2 t+r-n+3}\right)+t+r-n+2 \leq i \leq r-1 \\
& \text { and } 5 \leq l_{t} \leq \Delta_{t}-7 \\
n+i-(t+r+2) & \text { if } 2 t+r-n+5 \leq i \leq r-1 \\
& \text { and } \Delta_{t}-6 \leq l_{t} \leq \Delta_{t}\end{cases} \\
& f_{l}\left(v_{i}\right)= \begin{cases}\frac{i-r+1}{2} & \text { if } i \text { is odd and } r \leq i \leq n-1 \\
n+1-\left(\frac{i-r}{2}\right) & \text { if } i \text { is even and } r \leq i \leq n-1\end{cases} \\
& f_{l}\left(v_{n}\right)= \begin{cases}t+3 & \text { if } l_{t}=1,2 \\
t+2 & \text { if } 3 \leq l_{t} \leq \Delta_{t} .\end{cases}
\end{aligned}
$$


Then

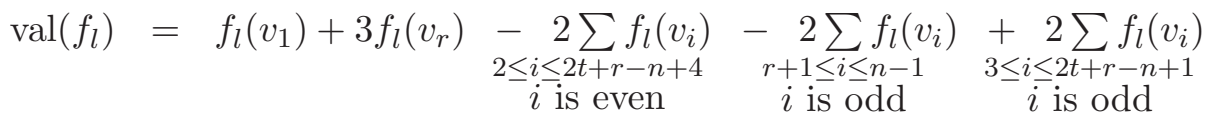

$$
\begin{aligned}
& \begin{aligned}
+2 f_{l}\left(v_{2 t+r-n+3}\right)+\underset{r+2 \leq i \leq n-2}{2} f_{l}\left(v_{i}\right) \\
i \text { is even }
\end{aligned} \\
& =2 n t+n-2 t^{2}-1+f_{l}\left(v_{1}\right)+2 f_{l}\left(v_{2 t+r-n+3}\right)=(2 n-2)+l \text {. }
\end{aligned}
$$

VI. For $\sum_{i=0}^{\frac{n}{2}-3} \Delta_{i}+1 \leq l \leq \sum_{i=0}^{\frac{n}{2}-2} \Delta_{i}$, observe that $\Delta_{\frac{n}{2}-2}=7$. We define a $\gamma$-labeling $f_{l}$ of $C_{n}+v_{1} v_{r}$ by

$$
\begin{aligned}
& f_{l}\left(v_{1}\right)= \begin{cases}\frac{n}{2}-l_{t} & \text { if } l_{t}=1,2,3 \\
\frac{n}{2}+4-l_{t} & \text { if } l_{t}=4,5,6,7\end{cases} \\
& f_{l}\left(v_{2}\right)= \begin{cases}0 & \text { if } l_{t}=2,5 \\
\frac{n}{2}-2 & \text { if } l_{t}=1,3 \\
\frac{n}{2}-1 & \text { if } l_{t}=4,6,7\end{cases} \\
& f_{l}\left(v_{3}\right)= \begin{cases}0 & \text { if } l_{t}=1,4 \\
\frac{n}{2}-1 & \text { if } l_{t}=2,3 \\
\frac{n}{2} & \text { if } l_{t}=5,6,7\end{cases} \\
& f_{l}\left(v_{i}\right)=\frac{n+4+r-i}{2} \quad \text { if } i \text { is even and } 4 \leq i \leq r-2 \\
& f_{l}\left(v_{i}\right)=n+1+\left(\frac{i-r}{2}\right) \text { if } i \text { is even and } r \leq i \leq n \\
& f_{l}\left(v_{i}\right)= \begin{cases}\frac{n}{2} & \text { if } i=5 \text { and } l_{t}=1,2,3 \\
\frac{i-5}{2} & \text { if } i \text { is odd, } 7 \leq i \leq n-3 \text { and } l_{t}=1,2,3 \\
\frac{i-3}{2} & \text { if } i \text { is odd, } 5 \leq i \leq n-3 \text { and } l_{t}=4,5,6 \\
\frac{i-3}{2} & \text { if } i \text { is odd, } 5 \leq i \leq n-5 \text { and } l_{t}=7 \\
0 & \text { if } i=n-3 \text { and } l_{t}=7\end{cases}
\end{aligned}
$$


$f_{l}\left(v_{n-1}\right)= \begin{cases}0 & \text { if } l_{t}=3,6 \\ \frac{n}{2}-3 & \text { if } l_{t}=1,2 \\ \frac{n}{2}-2 & \text { if } l_{t}=4,5,7\end{cases}$

Since $l_{t}=l-\sum_{i=0}^{\frac{n}{2}-3} \Delta_{i}=l-\frac{n^{2}}{2}-\frac{n}{2}+7$, it follows that for each $l$ with $\sum_{i=0}^{\frac{n}{2}-3} \Delta_{i}+1 \leq l \leq \sum_{i=0}^{\frac{n}{2}-2} \Delta_{i}, \operatorname{val}\left(f_{l}\right)=\frac{n^{2}}{2}+\frac{5 n}{2}-9+l_{t}=(2 n-2)+l$.

VII. For $\sum_{i=0}^{\frac{n}{2}-2} \Delta_{i}+1 \leq l \leq \sum_{i=0}^{\frac{n}{2}-1} \Delta_{i}$, define a $\gamma$-labeling $f_{l}$ of $C_{n}+v_{1} v_{r}$ by $f_{l}\left(v_{1}\right)= \begin{cases}\frac{n}{2}-1-l_{t} & \text { if } 1 \leq l_{t} \leq 3 \\ \frac{n}{2}+3-l_{t} & \text { if } 4 \leq l_{t} \leq \Delta_{\frac{n}{2}-1}\end{cases}$
$f_{l}\left(v_{2}\right)= \begin{cases}\frac{n}{2} & \text { if } 1 \leq l_{t} \leq 3 \\ \frac{n}{2}+2 & \text { if } 4 \leq l_{t} \leq \Delta_{\frac{n}{2}-1}\end{cases}$

$f_{l}\left(v_{i}\right)=\frac{i-1}{2} \quad$ if $i$ is odd, $3 \leq i \leq n-1$ and $i \neq 2 f_{l}\left(v_{1}\right)+1$

$f_{l}\left(v_{2 f_{l}\left(v_{1}\right)+1}\right)=0$

$f_{l}\left(v_{i}\right)= \begin{cases}\frac{n+4+r-i}{2} & \text { if } i \text { is even, } 4 \leq i \leq r-2 \text { and } 1 \leq l_{t} \leq 3 \\ \frac{n+2+r-i}{2} & \text { if } i \text { is even, } 4 \leq i \leq r-2 \text { and } 4 \leq l_{t} \leq \Delta_{\frac{n}{2}}-1\end{cases}$

$f_{l}\left(v_{i}\right)=n+1-\left(\frac{r-i}{2}\right)$ if $i$ is even and $r \leq i \leq n$.

Then

$$
\begin{aligned}
& \operatorname{val}\left(f_{l}\right)=-3 f_{l}\left(v_{1}\right)+2 f_{l}\left(v_{2}\right)+3 f_{l}\left(v_{r}\right)-\underset{\substack{3 \leq i \leq n-1 \\
i \text { is odd }}}{2 \sum} f_{l}\left(v_{i}\right)+\underset{\substack{4 \leq i \leq r-2 \\
\text { is even }}}{2 \sum_{l} f_{l}\left(v_{i}\right)} \\
& +\underset{r+2 \leq i \leq n}{2 \sum_{l}\left(v_{i}\right)} \\
& =-\frac{n^{2}}{4}+\frac{3 n}{2}+1-f_{l}\left(v_{1}\right)+2 f_{l}\left(v_{2}\right)+\underset{4 \leq i \leq n}{2 \sum_{i \leq i} f}\left(v_{i}\right) \\
& =(2 n-2)+l . \\
& i \text { is even }
\end{aligned}
$$

We now illustrate the proof of Proposition 2.2. The table 1 shows all variables in the proof of Proposition 2.2 that we use to find $\operatorname{spec}\left(C_{8}+v_{1} v_{4}\right)$. 


\begin{tabular}{|c|c|c|c|c|c|c|c|c|c|c|}
\hline & \multicolumn{5}{|c|}{$\operatorname{val}\left(f_{l}\right)=(2 n-2)+l$ of $C_{8}+v_{1} v_{4} \in$} & \multicolumn{3}{|c|}{$2 n-1, \frac{n^{2}+6 n+2}{2}$} & \multicolumn{2}{|c|}{$=[15,57]$} \\
\hline \multicolumn{11}{|c|}{$\Delta_{0}=2 n+2=18,1 \leq l \leq 18$} \\
\hline $\bar{l}$ & & $\gamma$-labeling $f_{l}: v_{1}$ & $v_{2}$ & $v_{3}$ & $v_{4}$ & $v_{5}$ & $v_{6}$ & $v_{7}$ & $v_{8}$ & $\operatorname{val}\left(f_{l}\right)$ \\
\hline 1 & & $\overline{2}$ & 1 & $\overline{0}$ & $\overline{3}$ & $\overline{4}$ & 5 & $\overline{6}$ & $\overline{7}$ & 15 \\
\hline 2 & & 1 & 0 & 2 & 3 & 4 & 5 & 6 & 7 & 16 \\
\hline 3 & & 3 & 1 & 0 & 4 & 5 & 6 & 7 & 8 & 17 \\
\hline 4 & & 2 & 1 & 0 & 4 & 5 & 6 & 7 & 8 & 18 \\
\hline 5 & & 4 & 1 & 0 & 5 & 6 & 7 & 8 & 9 & 19 \\
\hline 6 & & 3 & 1 & 0 & 5 & 6 & 7 & 8 & 9 & 20 \\
\hline 7 & & 2 & 1 & 0 & 5 & 6 & 7 & 8 & 9 & 21 \\
\hline 8 & & 1 & 0 & 2 & 5 & 6 & 7 & 8 & 9 & 22 \\
\hline 9 & & $\overline{3}$ & $\overline{0}$ & 1 & $\overline{6}$ & $\overline{5}$ & $\overline{7}$ & $\overline{8}$ & $\overline{9}$ & 23 \\
\hline 10 & & 2 & 0 & 1 & 6 & 5 & 7 & 8 & 9 & 24 \\
\hline 11 & & 3 & 0 & 2 & 6 & 7 & 8 & 9 & 1 & 25 \\
\hline 12 & & 4 & 0 & 2 & 6 & 7 & 8 & 9 & 1 & 26 \\
\hline 13 & & 4 & 0 & 3 & 7 & 8 & 9 & 1 & 2 & 27 \\
\hline 14 & & 5 & 0 & 3 & 7 & 8 & 9 & 1 & 2 & 28 \\
\hline 15 & & 5 & 0 & 4 & 8 & 9 & 1 & 2 & 3 & 29 \\
\hline 16 & & 6 & 0 & 4 & 8 & 9 & 1 & 2 & 3 & 30 \\
\hline 17 & & 6 & 0 & 5 & 9 & 1 & 2 & 3 & 4 & 31 \\
\hline 18 & & 7 & 0 & 5 & 9 & 1 & 2 & 3 & 4 & 32 \\
\hline \multicolumn{11}{|c|}{$\Delta_{1}=2 n-4(1)-1=11,19 \leq l \leq 29$} \\
\hline 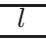 & $l_{1}$ & $\gamma$-labeling $f_{l}: v_{1}$ & $\overline{v_{2}}$ & $v_{3}$ & $v_{4}$ & $v_{5}$ & $v_{6}$ & $v_{7}$ & $\overline{v_{8}}$ & val $\left(f_{l}\right)$ \\
\hline$\overline{19}$ & 1 & $\overline{6}$ & $\overline{0}$ & $\overline{5}$ & $\overline{9}$ & $\overline{1}$ & $\overline{3}$ & $\overline{2}$ & $\overline{4}$ & $\overline{33}$ \\
\hline 20 & 2 & 7 & 0 & 5 & 9 & 1 & 3 & 2 & 4 & 34 \\
\hline 21 & 3 & 6 & 0 & 5 & 9 & 1 & 4 & 2 & 3 & 35 \\
\hline 22 & 4 & 7 & 0 & 5 & 9 & 1 & 4 & 2 & 3 & 36 \\
\hline 23 & 5 & 6 & 0 & 4 & 9 & 1 & 5 & 2 & 3 & 37 \\
\hline 24 & 6 & 7 & 0 & 4 & 9 & 1 & 5 & 2 & 3 & 38 \\
\hline 25 & 7 & 8 & 0 & 4 & 9 & 1 & 5 & 2 & 3 & 39 \\
\hline 26 & 8 & 5 & 0 & 4 & 9 & 1 & 7 & 2 & 3 & 40 \\
\hline 27 & 9 & 6 & 0 & 4 & 9 & 1 & 7 & 2 & 3 & 41 \\
\hline 28 & 10 & 5 & 0 & 4 & 9 & 1 & 8 & 2 & 3 & 42 \\
\hline 29 & 11 & 6 & 0 & 4 & 9 & 1 & 8 & 2 & 3 & 43 \\
\hline \multicolumn{11}{|c|}{$\Delta_{2}=2 n-4(2)-1=7,30 \leq l \leq 36$} \\
\hline $\bar{l}$ & $\bar{l}_{2}$ & $\gamma$-labeling $f_{l}: v_{1}$ & $v_{2}$ & $v_{3}$ & $v_{4}$ & $v_{5}$ & $v_{6}$ & $v_{7}$ & $v_{8}$ & $\operatorname{val}\left(f_{l}\right)$ \\
\hline 30 & 1 & 3 & 2 & $\overline{0}$ & 9 & $\overline{4}$ & 8 & 1 & 7 & $\overline{44}$ \\
\hline 31 & 2 & 2 & 0 & 3 & 9 & 4 & 8 & 1 & 7 & 45 \\
\hline 32 & 3 & 1 & 2 & 3 & 9 & 4 & 8 & 0 & 7 & 46 \\
\hline 33 & 4 & 4 & 3 & 0 & 9 & 1 & 8 & 2 & 7 & 47 \\
\hline 34 & 5 & 3 & 0 & 4 & 9 & 1 & 8 & 2 & 7 & 48 \\
\hline 35 & 6 & 2 & 3 & 4 & 9 & 1 & 8 & 0 & 7 & 49 \\
\hline 36 & 7 & 1 & 3 & 4 & 9 & 0 & 8 & 2 & 7 & 50 \\
\hline \multicolumn{11}{|c|}{$\Delta_{3}=\frac{n}{2}+3=7,37 \leq l \leq 43$} \\
\hline $\bar{l}$ & $l_{3}$ & $\gamma$-labeling $f_{l}: v_{1}$ & $v_{2}$ & $v_{3}$ & $v_{4}$ & $v_{5}$ & $v_{6}$ & $v_{7}$ & $v_{8}$ & $\operatorname{val}\left(f_{l}\right)$ \\
\hline 37 & 1 & 2 & 4 & 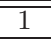 & 9 & 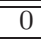 & 8 & 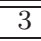 & 7 & 51 \\
\hline 38 & 2 & 1 & 4 & 0 & 9 & 2 & 8 & 3 & 7 & 52 \\
\hline 39 & 3 & 0 & 4 & 1 & 9 & 2 & 8 & 3 & 7 & 53 \\
\hline 40 & 4 & 3 & 6 & 1 & 9 & 2 & 8 & 0 & 7 & 54 \\
\hline 41 & 5 & 2 & 6 & 1 & 9 & 0 & 8 & 3 & 7 & 55 \\
\hline 42 & 6 & 1 & 6 & 0 & 9 & 2 & 8 & 3 & 7 & 56 \\
\hline 43 & 7 & 0 & 6 & 1 & 9 & 2 & 8 & 3 & 7 & 57 \\
\hline
\end{tabular}

Table 1: $\operatorname{spec}\left(C_{8}+v_{1} v_{4}\right)$ 
Proposition 2.3. For every even integer $n \geq 4$,

$$
\begin{aligned}
\operatorname{spec}\left(C_{n}+e\right) & =\left[\operatorname{val}_{\min }\left(C_{n}+e\right), \mathrm{val}_{\max }\left(C_{n}+e\right)\right] \\
& = \begin{cases}{\left[2 n-1, \frac{n^{2}+5 n-2}{2}\right]} & \text { if } n=4,6 \\
{\left[2 n-1, \frac{n^{2}+6 n-10}{2}\right]} & \text { if } n \geq 8\end{cases}
\end{aligned}
$$

where $e$ is a chord joining two vertices with even distance in even cycle $C_{n}$.

Proof. Assume by (1) that $C_{n}+e=C_{n}+v_{1} v_{r}$ where $3 \leq r \leq \frac{n}{2}+1$ and $r$ is odd.

First we consider $C_{n}+v_{1} v_{r}$ when $n \geq 8$. For each integer $i$ with $0 \leq i \leq$ $\frac{n}{2}-1$, let

$$
\Delta_{i}= \begin{cases}2 n+2 & \text { if } i=0 \\ 2 n-4 i-1 & \text { if } 1 \leq i \leq \frac{n}{2}-2 \\ \frac{n}{2}-3 & \text { if } i=\frac{n}{2}-1\end{cases}
$$

For each integer $l$ with

$$
1 \leq l \leq \sum_{i=0}^{\frac{n}{2}-1} \Delta_{i}=\left|\left[\operatorname{val}_{\text {min }}\left(C_{n}+v_{1} v_{r}\right), \operatorname{val}_{\text {max }}\left(C_{n}+v_{1} v_{r}\right)\right]\right|,
$$

we show that there is a $\gamma$-labeling $f_{l}$ whose value is $(2 n-2)+l$.

1. For $1 \leq l \leq \Delta_{0}=2 n+2$, a $\gamma$-labeling $f_{l}$ is defined in similar way as described in I., II., and III. of Proposition 2.2.

Now we consider $\Delta_{0}+1 \leq l \leq \sum_{i=0}^{\frac{n}{2}-1} \Delta_{i}$, by letting $\left(t, l_{t}\right)$ be a pair of integers with $1 \leq t \leq \frac{n}{2}-1$ and $l_{t}=l-\sum_{i=0}^{t-1} \Delta_{i}$, that is, $1 \leq l_{t} \leq \Delta_{t}$. A $\gamma$-labeling $f_{l}$ can be constructed as the following procedure:

2. For $\Delta_{0}+1 \leq l \leq \sum_{i=0}^{\frac{n-r-1}{2}} \Delta_{i}$ of $C_{n}+v_{1} v_{r}$ by

$$
f_{l}\left(v_{1}\right)= \begin{cases}n-(t-1) & \text { if } l_{t}=\Delta_{t}-4 \\ n-t & \text { if } l_{t} \text { is even and } l_{t} \neq \Delta_{t}-3, \Delta_{t}-1 \\ n-(t+1) & \text { if } l_{t} \text { is odd and } l_{t} \neq \Delta_{t}-4 \\ n-(t+2) & \text { if } l_{t}=\Delta_{t}-3, \Delta_{t}-1\end{cases}
$$




$$
\begin{aligned}
& f_{l}\left(v_{2}\right)=0 \\
& f_{l}\left(v_{i}\right)= \begin{cases}n+i-(t+r+1) & \text { if } 3 \leq i \leq r-1 \text { and } 1 \leq l_{t} \leq \Delta_{t}-(2 r-1) \\
n+i-(t+r+2) & \text { if } 3 \leq i \leq f_{l}\left(v_{2 t+r}\right)+t+r-n+1 \\
& \text { and } \Delta_{t}-(2 r-2) \leq l_{t} \leq \Delta_{t}-7 \\
n+i-(t+r+1) & \text { if } f_{l}\left(v_{2 t+r}\right)+t+r-n+2 \leq i \leq r-1 \\
& \text { and } \Delta_{t}-(2 r-2) \leq l_{t} \leq \Delta_{t}-7 \\
n+i-(t+r+2) & \text { if } 3 \leq i \leq r-1 \text { and } \Delta_{t}-6 \leq l_{t} \leq \Delta_{t}\end{cases} \\
& f_{l}\left(v_{i}\right)= \begin{cases}\frac{i-r+1}{2} & \text { if } i \text { is even and } r \leq i \leq 2 t+r+1 \\
n+1-\left(\frac{i-r}{2}\right) & \text { if } i \text { is odd and } r \leq i \leq 2 t+r-2\end{cases} \\
& f_{l}\left(v_{2 t+r}\right)= \begin{cases}\left\lceil\frac{l_{t}+2}{2}\right\rceil+t & \text { if } 1 \leq l_{t} \leq \Delta_{t}-5 \\
\left\lceil\frac{l_{t}}{2}\right\rceil+t & \text { if } l_{t}=\Delta_{t}-4 \\
\left\lceil\frac{l_{t}+3}{2}\right\rceil+t & \text { if } \Delta_{t}-3 \leq l_{t} \leq \Delta_{t}\end{cases} \\
& f_{l}\left(v_{i}\right)= \begin{cases}i-(t+r-1) & \text { if } 2 t+r+2 \leq i \leq n \text { and } l_{t}=1,2 \\
i-(t+r) & \text { if } 2 t+r+2 \leq i \leq f_{l}\left(v_{2 t+r}\right)+t+r-1 \\
& \text { and } 3 \leq l_{t} \leq \Delta_{t}-(2 r+1) \\
i-(t+r-1) & \text { if } f_{l}\left(v_{2 t+r}\right)+t+r \leq i \leq n \\
& \text { and } 3 \leq l_{t} \leq \Delta_{t}-(2 r+1) \\
i-(t+r) & \text { if } 2 t+r+2 \leq i \leq n \text { and } \Delta_{t}-2 r \leq l_{t} \leq \Delta_{t} .\end{cases}
\end{aligned}
$$

Then

$$
\begin{aligned}
& \left.\operatorname{val}\left(f_{l}\right)=f_{l}\left(v_{1}\right)-2 f_{l}\left(v_{2}\right)+3 f_{l}\left(v_{r}\right) \underset{r+1 \leq i \leq 2 t+r+1}{i \text { is even }} \underset{\substack{r+2 \leq i \leq 2 t+r-2 \\
i \text { is odd }}}{2} f_{l}\left(v_{i}\right)+2 f_{l}\left(v_{i}\right)+2 t+r\right) \\
& =2 n t+n-2 t^{2}-1+f_{l}\left(v_{1}\right)+2 f_{l}\left(v_{2 t+r}\right)=(2 n-2)+l \text {. }
\end{aligned}
$$

3. For $\sum_{i=0}^{\frac{n-r-1}{2}} \Delta_{i}+1 \leq l \leq \sum_{i=0}^{\frac{n}{2}-2} \Delta_{i}$, define a $\gamma$-labeling $f_{l}$ of $C_{n}+v_{1} v_{r}$ by 


$$
\begin{aligned}
& f_{l}\left(v_{1}\right)= \begin{cases}n-(t-1) & \text { if } l_{t}=\Delta_{t}-4 \\
n-t & \text { if } l_{t} \text { is even and } l_{t} \neq \Delta_{t}-3, \Delta_{t}-1 \\
n-(t+1) & \text { if } l_{t} \text { is odd and } l_{t} \neq \Delta_{t}-4 \\
n-(t+2) & \text { if } l_{t}=\Delta_{t}-3, \Delta_{t}-1\end{cases} \\
& f_{l}\left(v_{2}\right)=0 \\
& f_{l}\left(v_{i}\right)= \begin{cases}\frac{n-1+i-r}{2} & \text { if } i \text { is even and } 3 \leq i \leq 2 t+r-n+3 \\
\frac{n+4+r-i}{2} & \text { if } i \text { is odd and } 3 \leq i \leq 2 t+r-n\end{cases} \\
& f_{l}\left(v_{2 t+r-n+2}\right)= \begin{cases}\left\lceil\frac{l_{t}+2}{2}\right\rceil+t & \text { if } 1 \leq l_{t} \leq \Delta_{t}-5 \\
\left\lceil\frac{l_{t}}{2}\right\rceil+t & \text { if } l_{t}=\Delta_{t}-4 \\
\left\lceil\frac{l_{t}+3}{2}\right\rceil+t & \text { if } \Delta_{t}-3 \leq l_{t} \leq \Delta_{t}\end{cases} \\
& \left\{\begin{aligned}
n+i-(t+r+1) & \text { if } 2 t+r-n+4 \leq i \leq r-1 \text { and } l_{t}=1,2 \\
n+i-(t+r+2) & \text { if } 2 t+r-n+4 \leq i \leq f_{l}\left(v_{2 t+r-n+2}\right) \\
& +t+r-n+1
\end{aligned}\right. \\
& f_{l}\left(v_{i}\right)=\left\{\begin{aligned}
& \text { and } 3 \leq l_{t} \leq \Delta_{t}-r \\
n+i-(t+r+1) & \text { if } f_{l}\left(v_{2 t+r-n+2}\right)+t+r-n+2 \leq i \leq r-1 \\
& \text { and } 3 \leq l_{t} \leq \Delta_{t}-r
\end{aligned}\right. \\
& n+i-(t+r+2) \text { if } 2 t+r-n+4 \leq i \leq r-1 \\
& \text { and } \Delta_{t}-(r-1) \leq l_{t} \leq \Delta_{t}
\end{aligned}
$$

$$
f_{l}\left(v_{i}\right)= \begin{cases}\frac{i-r+1}{2} & \text { if } i \text { is even and } r \leq i \leq n \\ n+1-\left(\frac{i-r}{2}\right) & \text { if } i \text { is odd and } r \leq i \leq n .\end{cases}
$$

Then

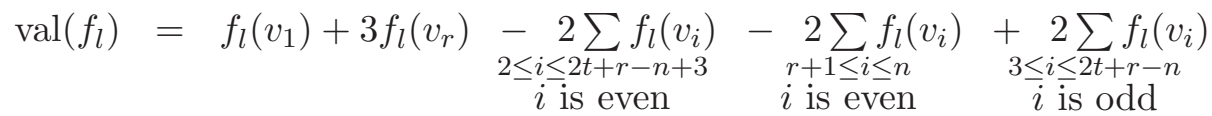

$$
\begin{aligned}
& =2 n t+n-2 t^{2}-1+f_{l}\left(v_{1}\right)+2 f_{l}\left(v_{2 t+r-n+2}\right)=(2 n-2)+l \text {. }
\end{aligned}
$$

4. For $\sum_{i=0}^{\frac{n}{2}-2} \Delta_{i}+1 \leq l \leq \sum_{i=0}^{\frac{n}{2}-1} \Delta_{i}$, define a $\gamma$-labeling $f_{l}$ of $C_{n}+v_{1} v_{r}$ 
by

$$
\begin{array}{rlrl}
f_{l}\left(v_{1}\right) & =\frac{n}{2}-3-l_{t} & \\
f_{l}\left(v_{2}\right) & =\frac{n}{2} & & \\
f_{l}\left(v_{i}\right) & =\frac{n+4+r-i}{2} & & \text { if } i \text { is odd, } 3 \leq i \leq r-2 \\
f_{l}\left(v_{i}\right) & =\frac{i}{2}-1 & & \text { if } i \text { is even, } 4 \leq i \leq n \\
f_{l}\left(v_{2 f_{l}\left(v_{1}\right)+2}\right) & =0 & & \text { and } i \neq 2 f_{l}\left(v_{1}\right)+2 \\
f_{l}\left(v_{i}\right) & =n+1+\frac{r-i}{2} & & \text { if } i \text { is odd, } r \leq i \leq n-1 .
\end{array}
$$

Then

$$
\begin{aligned}
\operatorname{val}\left(f_{l}\right)= & -3 f_{l}\left(v_{1}\right)+3 f_{l}\left(v_{r}\right)+\underset{3 \leq i \leq r-2}{2 \sum_{i} f_{l}\left(v_{i}\right)} \underset{\substack{r+2 \leq i \leq n-1 \\
i \text { is odd }}}{i \text { is odd }} \underset{\substack{2 \sum_{i \leq n-2} \\
i \text { is even }}}{2 \sum_{l}\left(v_{i}\right)} f_{l}\left(v_{i}\right) \\
= & \frac{n^{2}}{2}+\frac{5 n}{2}-2+l-\frac{n^{2}}{2}-\frac{n}{2}=(2 n-2)+l .
\end{aligned}
$$

Next, we consider $C_{4}+v_{1} v_{3}$, by letting $\Delta_{0}=10$ and $\Delta_{1}=1$. Therefore $\Delta_{0}+\Delta_{1}=\left|\left[\operatorname{val}_{\min }\left(C_{4}+v_{1} v_{3}\right), \operatorname{val}_{\max }\left(C_{4}+v_{1} v_{3}\right)\right]\right|$.

If $1 \leq l \leq \Delta_{0}=10$, then we define a $\gamma$-labeling $f_{l}$ similar to $\gamma$-labeling $f_{l}$ in 1. of $C_{n}+v_{1} v_{r}$ when $n \geq 8$.

Otherwise, if $l=\Delta_{0}+\Delta_{1}=11$, then a $\gamma$-labeling $f_{l}$ of $C_{4}+v_{1} v_{3}$ defined by $f_{l}\left(v_{1}\right)=4, f_{l}\left(v_{2}\right)=0, f_{l}\left(v_{3}\right)=5$, and $f_{l}\left(v_{4}\right)=1$ has $\operatorname{val}\left(f_{l}\right)=(2 n-2)+l$.

Last, we consider $C_{6}+v_{1} v_{3}$, by letting $\Delta_{0}=14, \Delta_{1}=7$ and $\Delta_{2}=1$. Then $\Delta_{0}+\Delta_{1}+\Delta_{2}=\left|\left[\operatorname{val}_{\min }\left(C_{6}+v_{1} v_{3}\right), \operatorname{val}_{\max }\left(C_{6}+v_{1} v_{3}\right)\right]\right|$.

If $1 \leq l \leq \Delta_{0}+\Delta_{1}=21$, then we define a $\gamma$-labeling $f_{l}$ similar to $\gamma$-labeling $f_{l}$ in 1. of $C_{n}+v_{1} v_{r}$ when $n \geq 8$.

Otherwise, if $l=\Delta_{0}+\Delta_{1}+\Delta_{2}=22$, then define a $\gamma$-labeling $f_{l}$ of $C_{6}+v_{1} v_{3}$ by $f_{l}\left(v_{1}\right)=5, f_{l}\left(v_{2}\right)=0, f_{l}\left(v_{3}\right)=7, f_{l}\left(v_{4}\right)=1, f_{l}\left(v_{5}\right)=6$, and $f_{l}\left(v_{6}\right)=2$ having $\operatorname{val}\left(f_{l}\right)=(2 n-2)+l$.

Proposition 2.4. For every odd integer $n \geq 5$,

$$
\operatorname{spec}\left(C_{n}+e\right)=\left[\operatorname{val}_{\min }\left(C_{n}+e\right), \operatorname{val}_{\text {max }}\left(C_{n}+e\right)\right]=\left[2 n-1, \frac{n^{2}+6 n-3}{2}\right]
$$


The proof of Proposition 2.4 is similar to Propositions 2.2 and 2.3 and is therefore omitted.

\section{Final Remarks}

In this paper, we have established the $\gamma$-spectrum of cycle with one arbitrary chord. A natural question arises how to determine the extremal values and then $\gamma$-spectrum of cycle with two or more chords.

\section{Acknowledgments}

Research supported by Srinakharinwirot University, Year 2015.

\section{References}

[1] G. D. Bullington, L. L. Eroh, and S. J. Winters, $\gamma$-labelings of complete bipartite graphs, Discuss. Math. Graph Theory, 30 (2010), 45-54.

[2] G. Chartrand, D. Erwin, D.W. VanderJagt, P. Zhang, $\gamma$-labelings of graphs, Bull. Inst. Combin. Appl., 44 (2005), 51-68.

[3] G. Chartrand, D. Erwin, D.W. VanderJagt, P. Zhang, On $\gamma$-labelings of trees, Discuss. Math. Graph Theory, 25, No. 3 (2005), 363-383.

[4] G. Chartrand, P. Zhang, Introduction to Graph Theory, The Walter Rudin student series in advanced mathematics, McGraw-Hill Higher Education, Boston (2005).

[5] C.M. da Fonseca, V. Saenpholphat, and P. Zhang, Extremal values for a $\gamma$-labeling of a cycle with a triangle, Utilitas Math., 92 (2013), 167-185.

[6] C.M. da Fonseca, V. Saenpholphat, P. Zhang, On $\gamma$-labelings of graphs, Utilitas Math., to appear.

[7] C.M. da Fonseca, V. Saenpholphat, P. Zhang, The $\gamma$-spectrum of a graph, Ars Combin., 101 (2011), 109-127.

[8] J.A. Gallian, A dynamic survey of graph labeling, Electron. J. Combin. DS6, 16 (2009), 1-219. 
[9] S.W. Golomb, How to number a graph, In: Graph Theory and Computing, Academic Press, New York (1972).

[10] V. Khemmani, S. Saduakdee, $\gamma$-labeling of cycle with one chord, Lecture Notes in Computer Science, submitted.

[11] A. Rosa, On certain valuations of the vertices of a graph, In: Theory of Graphs (Internat. Sympos., Rome, 1966), Gordon and Breach, New York; Dunod, Paris (1966). 